\title{
La Movilización del Conocimiento en Tres Tiempos
}

\author{
Judith Naidorf \& Mauro Alonso
}

\begin{abstract}
La movilización del conocimiento en tres tiempos: antes ¿qué investigar? ¿por qué? ¿para quién?, durante ¿cómo me evalúan? ¿qué ponderan? ¿cómo oriento mis acciones para ser evaluado favorablemente? y después ¿quién usa mi conocimiento? ¿a quién sirve? Las ciencias se han ocupado siempre de estar orientadas a la solución de problemas. Esto se ha tornado más o menos cierto según los temas, modalidades, mediaciones en el proceso de intervención en la realidad a partir de la evidencia empírica que arrojan los resultados de las investigaciones científicas. Tanto las denominadas ciencia básica y aplicada poseen un fin, un norte, una orientación que supera la clásica caracterización de "ampliación de las fronteras de las disciplinas" o "promoción general del conocimiento". La definición de las agendas de investigación siempre supera la mera curiosidad y es influenciada por múltiples factores que inciden en ella: la relevancia social de un tema, la medición del impacto de una determinada acción humana o una política pública, el financiamiento público o privado que pueda obtenerse, la búsqueda de comprensión de aspectos particulares de la realidad presente o de la historia y también su orientación hacia la transformación de aquello que se estudia. En esta línea argumental la movilización del conocimiento se concibe como aquel campo de investigaciones que se esfuerza por promover el uso del conocimiento científico. En efecto, la movilización del conocimiento supone una doble concepción de la producción de conocimiento: por un lado asume como indispensable el uso del conocimiento producido y por tanto imbrica el uso como parte del proceso de producción de conocimiento a la vez que lo reconoce como tarea del investigador y de la investigadora.
\end{abstract}

Palabras Clave:

movilización del conocimiento; agendas de investigación; evaluación académica; utilidad del conocimiento 


\title{
A Mobilização do conhecimento em três tempos
}

Resumo: A Mobilização do conhecimento em três tempos. Antes, o quê investigar? Porquê? Para quem?, Durante? Como serei avaliado? Que critérios? Como posso orientar as minhas ações para ser avaliado favoravelmente? E depois, quem usa o conhecimento? Para que serve? As ciências sempre se voltaram para a resolução de problemas. Esta ideia tornou-se mais ou menos verdade dependendo do assunto, das modalidades e das mediações no processo de intervenção na realidade a partir das evidências empíricas que trazem os resultados da investigação científica. Tanto a chamada ciência básica como a aplicada tem um fim, uma orientação, um norte que vai além da caracterização clássica de "expandir as fronteiras das disciplinas" ou da "promoção geral do conhecimento." A definição de agendas de investigação supera sempre a mera curiosidade e é influenciada por muitos fatores: a relevância social de um tema, a medição do impacto de uma determinada ação humana ou uma política pública, o financiamento público ou privado que pode ser obtido, a busca pela compreensão de aspectos particulares da realidade presente ou histórica, e também a orientação para a transformação daquilo que se estuda. Nesta linha de argumentação, a mobilização do conhecimento é concebida como aquele campo de pesquisa que se esforça por promover o uso do conhecimento científico. Com efeito, a mobilização do conhecimento supõe uma dupla concepção da produção de conhecimento: por um lado, assume como indispensável o uso do conhecimento produzido e, portanto, entrelaça o uso como parte do processo de produção de conhecimento e, por outro, reconhece-o como tarefa do investigador e da investigadora.

Palavras-chave: mobilização do conhecimento: agendas de investigação; avaliação académica; utilidade do conhecimento

\section{Mobilization of Knowledge in three times}

\begin{abstract}
Mobilization of Knowledge in three times. Before, What to research? Why? For Whom?, During, How will I be evaluated? Under what considerations? How to guide my actions in order to be favourably evaluated?, Finally, Who uses my knowledge? To whom it serves? The sciences have always been concerned with contributing to solving social problems and demands. This has become more or less true according to the topics, modalities, mediations in the intervention process that the results of the scientific investigations show. Both the so-called basic and applied science have an end, a north, and an orientation that goes beyond the classic characterization of "broadening the boundaries of each discipline" or the "general promotion of knowledge". The definition of research agendas always surpasses the mere curiosity and is influenced by multiple factors that influence it: the social relevance of a subject, the measurement of the impact of a given human action or a public policy, public or private financing that can be obtained, the search for understanding of particular aspects of the present reality or history and also its orientation towards the transformation of what is studied.In this line of argument knowledge mobilization is conceived as that field of research that strives to promote the use of scientific knowledge. The mobilization of knowledge presupposes a dual conception of the production of knowledge: on the one hand it assumes as indispensable the use of knowledge produced and therefore the use of than knowledge as part of the process of research.
\end{abstract}

Keywords: knowledge mobilization; research agendas; academic evaluation; knowledge utility.

\section{La mobilisation des connaissances en trois temps}

Résumé : La mobilisation de connaissance en trois temps. Avant, sur quoi on fait des recherches ? Pourquoi ? À qui ? Au cours ? Comment on sera-t-il évalué ? Quels critères ? Comment on peut guider ses actions afin d'être évalué favorablement? Et après, qui peut utiliser ma connaissance ? A quoi sert-il ? Les sciences ont toujours dû être axées sur la résolution de problème. Cela est devenu plus ou moins vrai selon le sujet, les modalités, les médiations dans le processus d'intervention dans la réalité de preuves empiriques qui jettent les résultats de la recherche scientifique. Les soi-disant sciences fondamentales et appliquées ont une fin, une orientation qui va au-delà de la caractérisation classique d' " élargir les frontières des disciplines " ou " promotion générale des connaissances». La définition des programmes de recherche dépasse toujours la simple curiosité et est influencée par de nombreux facteurs qui l'influencent : la pertinence sociale d'un sujet, la mesure de l'impact d'une action humaine ou une politique publique, le financement public ou privé peuvent être obtenus, la recherche de la compréhension des aspects particuliers de la réalité actuelle ou l'histoire et aussi son orientation vers la transformation de ce qui est étudié. Dans cette ligne de mobilisation du savoir, l'argument est conçu comme un domaine de recherche qui vise à promouvoir l'utilisation des connaissances scientifiques. En effet, la mobilisation des connaissances a une double conception de la production de la connaissance : on suppose aussi essentiel d'utiliser les connaissances produites et donc entremêle l'utilisation dans le cadre du processus de production de connaissances tout en reconnaissant comme tâche le chercheur et la chercheuse.

Mots-clés: mobilisation des connaissances; programmes de recherche; évaluation académique; utilité des connaissances. 


\section{La movilización del conocimiento: definiciones e implicancias}

Desde comienzos de siglo, surge para la discusión de las políticas de investigación en ciencias sociales y humanas el concepto de movilización del conocimiento como un ensayo de respuesta que permita enlazar con mayor grado de éxito la producción de conocimiento científico con su uso efectivo. El Consejo de Investigaciones en Ciencias Sociales y Humanidades de Canadá (SSHRC) define la movilización del conocimiento como:

"El flujo y consumo recíproco y complementario del conocimiento científico entre investigadores, mediadores e interlocutores y usuarios de dicho conocimiento-dentro y más allá de la academia-que procuralograr la maximización de los beneficios para los usuarios así como el logro de conocimientos creados en y paraCanadá y/o internacionalmente que provoquen consecuencias positivas y que por ultimo permita -ademásmejorar el perfil- en término de riqueza e impacto- de la investigación en ciencias sociales y humanidades"1

La movilización del conocimiento supone entonces una serie de estrategias, procesos, acciones que son identificadas en el quehacer cotidiano de los científicos y las científicas así como una serie de recomendaciones que la implican en su carácter normativo orientadas atender los procesos de vinculación entre productores y usuarios de conocimiento. Los múltiples trabajos publicados que mencionaremos a continuación -que son tanto estudios de caso como de debate teórico- ofrecen diagnósticos que se proponen ofrecer definiciones sobre: los actores que intervienen en la producción, las diferentes morfologías que adopta el conocimiento que se moviliza como así también los desafíos que presentan los canales de diálogo entre productores y usuarios del conocimiento denominado científico.

El concepto de movilización del conocimiento, entonces, asume diferentes perspectivas y definiciones: uso de la evidencia y del resultado de las investigaciones para la toma de decisiones en políticas públicas (Nutleyet. al, 2007); método o herramienta que facilita la traslación de resultados de la investigación a la acción (Bennetet. al, 2007); esfuerzos por compartir resultados de investigación con posibles usuarios (Levin, B., 2011); acciones que permiten dejar el conocimiento listo para la acción y su intervención mediante interlocutores (Levesque, 2009).

En términos cuantitativos, el campo de estudios sobre movilización del conocimiento -inaugurado a principios de este milenio- ha florecido resultando en una proliferación de publicaciones sobre el campo. En efecto, siguiendo la Figura 1, es posible observar el crecimiento exponencial de publicaciones sobre movilización del conocimiento 
indexadas en la base SCOPUS, especialmente luego del año 2008, llegando punto más alto en el año 2016 de 34 publicaciones:



FIGURA 1: Evolución de la cantidad de publicaciones sobre KMb indexados en SCOPUS2

En el plano cualitativo, mayormente, las publicaciones sobre la movilización de conocimiento ofrecen un estudio de caso, relevamiento empírico o evaluación de política pública del campo de la educación3. No nos detendremos in extenso en los distintos modelos resultantes de investigaciones empíricas del campo sino que intentaremos incluir algunas de las consideraciones que consideramos más relevantes y que suelen enfocarse en el análisis de las estrategias de "gestores del conocimiento"4 o Knowledge-brokeringentre investigadores y potenciales usuarios (Cooper y Shewchuk, 2015).

Kochanek, Scholz, yGarcia (2015) provee un modelo analítico para describir y promover estrategias de vinculación múltiples entre stakeholders e investigadores que consoliden sociedades de investigación (Kochanek et al, 2015).

Scott, Jabbar, LaLonde, DeBrayyLubienski(2015) por su parte, ponen el acento en el rol de organizaciones del tercer sector y muestran como estas organizaciones intermediarias que apelan a la movilización del conocimiento en educación (tanto financiadoras como demandantes y adoptantes de resultados de investigación) comienzan a mostrar cambios en las prácticas de actores del campo de la educación pública.

En este sentido, un rasgo común sobre el que las investigaciones ponen el acento resultan claramente sistematizados en el modelo de Teoría de la Acción de la Utilización del conocimiento descripta por Kochanek et al (2015) que hacen foco en la etapa 
de transmisión, transferencia del conocimiento y exploran estrategias para que el conocimiento producido sea efectivamente utilizado según lo descripto en la figura 1:

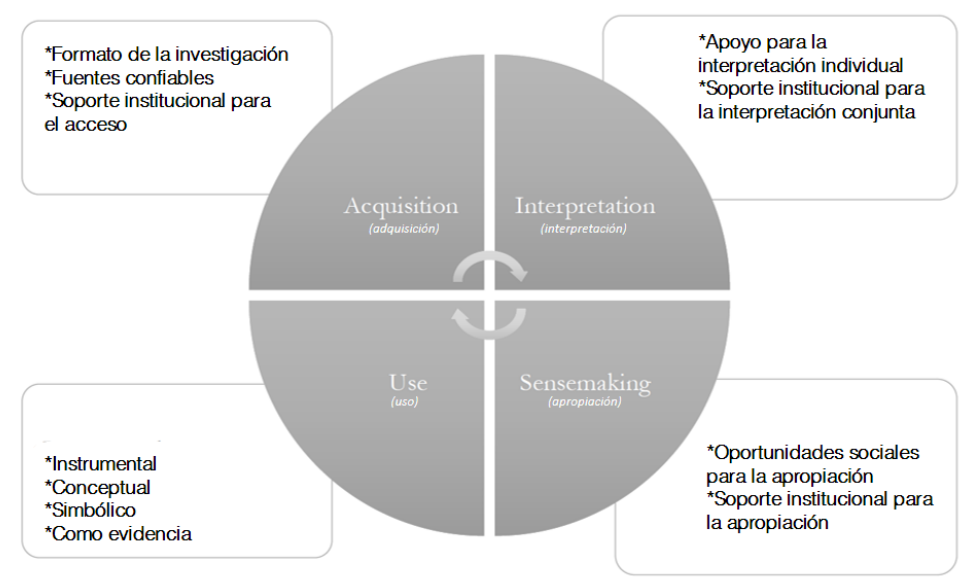

FIGURA 2: Teoría de la Acción de la Utilización del conocimiento5

En efecto, el modelo presentado describe las etapas del ciclo de utilización, comenzando en la adquisición, la interpretación y la apropiación (sensemaking) para luego consolidarse en uso efectivo. Las prácticas que este modelo describe se orientan a identificar y promover las capacidades necesarias de los actores sociales involucrados en el uso del conocimiento, puesto que "la utilización del conocimiento se sitúa en contextos sociales y organizacionales que influyen sobre las acciones individuales" (Kochanek et al, 2015).

Incluso reconociendo la importancia de la promoción de capacidades de apropiación de conocimiento, gran parte de la literatura considera que los procesos de producción y uso de conocimiento son un continuum olvidando una de las dimensiones de análisis: las prácticas de los investigadores y las investigadoras y los procesos que operan sobre estas. Los análisis sobre la movilización de conocimiento, incluso aquellos orientados al desarrollo de procesos de investigación colaborativa (CRP por sus siglas sajonas) que proponen integrar efectivamente practicioners (usuarios) en el proceso de producción de conocimiento (Burkhardt y Schoenfeld, 2003) no reparan en las dimensiones estructurales que operan sobre las prácticas de los científicxs en tanto que habilitan y constriñen los cursos de acción posibles de los investigadorxs, en especial en contextos periféricos en países en vías de desarrollo. Adicionalmente, 
en línea con algunas discusiones recientes respecto del lugar que ocupan los valores en la práctica científica, Da Silva (2016) propone reconsiderar el carácter socialmente construido y contingente de las categorías con las que, en especial los científicos sociales, piensan las sociedades contemporáneas.

La discusión sobre el aumento del uso efectivo del conocimiento científico pareciera confluir hacia la necesidad de reconocer a Ixsinterlocutorxs no-académicos como parte del proceso de producción de conocimiento incluso auncuando los modelos que intenten explicar estas vinculaciones no presenten respuestas definitivas. Esto pareciera ser uno de los principales méritos de los estudios de casos que caracterizan aquellos que se presentan en referencia a la movilización del conocimiento puesta en práctica. Sin embargo, consideramos que movilizar conocimiento, como propuesta normativa, no puede obviar aquellas limitantes estructurales que existen en la "cocina de la ciencia" sino que debe incorporarlas como variables que operan necesariamente sobre los procesos de movilización de conocimiento que se consideren exitosos.

Por su parte y a partir de un trabajo colectivo enmarcado en el Grupo de Trabajo de Clacso denominado "Ciencia social politizada y móvil en y para una agenda latinoamericana de investigaciones orientada desde la universidad" vigente de 2013 a 2016 hemos arribado a la siguiente definición:

"A diferencia de la difusión, donde no se implica, per se, compromiso alguno con la responsabilidad en el proceso que conduce hacia el canal de llegada del conocimiento elaborado, la movilidad o movilización del conocimiento involucra un paso intermedio entre el resultado arribado y su aplicación práctica. La metáfora utilizada para diferenciar difusión o diseminación, por un lado, de movilidad o movilización del conocimiento, por el otro, es la que se puede homologar al logro del crecimiento de una planta y la simple distribución de las semillas en una parcela de tierra. Mientras difusión o diseminación implican sólo arrojar las semillas a la tierra, la movilidad o movilización involucra otros procesos ligados al rastrillaje de la tierra: su fertilización, el riego, así como el compromiso de que -al final - lo plantado florezca.Con todo, hemos puesto a prueba y en tensión dicha categoría en congresos, conferencias y ante distintos interlocutores porque afirmamos su potencial como una categoría elaborada exclusivamente para las ciencias sociales.En estudios anteriores analizamos la categoría de transferencia del conocimiento (Naidorf, 2009) y transferencia social del conocimiento (Llomovatte, Naidorf y Pereyra, 2009), asumiendo - de modo particular - el uso que se ha hecho de la misma a partir de la conversión de la tradicional extensión universitaria en una amplia gama de actividades que van desde la venta de servicios a la transferencia 
tecnológica. Esta última categoría había sido elaborada para disciplinas tales como ingeniería, bioquímica y otras ciencias naturales, aspecto que limitaba el análisis sobre el papel de las ciencias sociales y su rol en un nuevo escenario que apela a la relevancia - en tanto adecuación de los propósitos de los proyectos - de las políticas públicas o de los objetivos estratégicos" (Naidorf y Perrotta, 2015: 28-29).

\section{La operacionalización de la movilización del conocimiento}

A partir de recuperar las consideraciones y definiciones presentadas y procurando ampliarlas y complejizarlas, nos proponemos operacionalizar el marco normativo acerca de la movilización del conocimiento en tres dimensiones que intervienen de forma activa sobre los procesos de producción de conocimiento y que ofrecen una forma de analizar la capacidad de movilización de conocimiento de la política pública en investigación científica: 1) la definición de agendas de investigación; 2) la evaluación de la actividad académica y 3) el uso del conocimiento producido. Presentaremos a continuación estas tres dimensiones en tanto que interconectadas y en conjunto nos permiten observar el proceso de movilización del conocimiento como así también Ixsactorxs que intervienen en la definición de cada una de ellas en sus componentes:

1. la definición de AGENDAS. En el estudio de las agendas se incluyen los análisis sobre las prioridades que establecen las políticas pública de los organismos nacionales y supranacionales -internacionales o regionales-, las influencias que los organismos de financiamiento público y privados ejercen sobre la elección de temas de investigación por parte de investigadores individuales (sean estos senior, junior o tesistas), equipos de investigación, institutos o centros de investigación, etc. Asimismo la definición de temas estratégicos, orientaciones y recomendaciones son objeto de estudio de esta dimensión de la movilización del conocimiento.

2. La característica que asume la EVALUACIÓN académica y del desempeño de IXsinvestigadorxs. En esa área se analiza el formateo de modalidades que asume el trabajo académico, tiempos y formas de investigar que afectan al propio objeto de estudio, a las relaciones académicas, al rol social del científico o de la científica y a su razón de ser, el perfil de investigador o investigadora que se promueve, la influencia en los modos de formación de los investigadores nóveles y las señales que emiten los sistemas de evaluación en tanto orientadora de actividad científica. Asimismo desde esta dimensión estudiamos las formas más o menos estandarizadas de evaluación académica, los criterios comunes y los específicos y diferenciados, los argumentos y críticas que motivan las discusiones en torno a ellos, las comparaciones entre países, 
las correcciones y sus justificaciones, las necesidades a las que responde la evaluación, etc.

3. El USO del conocimiento como aspecto en el que se entrecruzan su equívoca definición, su relación con la mediatez y la inmediatez del mismo, los tipos de "usuarios" sean estos agentes de la política pública que sustentan sus acciones a partir de la evidencia científica y a partir de resultados de investigaciones propias o generadas por la academia o por empresas privadas (consultoras) u otros actores sociales, la "usabilidad" como criterio de potencialidad previa a su puesta en práctica (Fischman, 2012), el involucramiento de actores "no científicos" en la creación del conocimiento científico, la relación uso-utilidad, etc.

A continuación se desarrollan cada una de las dimensiones in extenso lo que no hace más que abrir aún más la multiplicidad de temas que incumben a la movilización del conocimiento y a su potencial como analizador de las políticas de investigación así como también a las políticas universitarias y a las científicas en general y en particular.

\subsection{La implicación en la selección de temas de investigación: AGENDAS}

Cuando se estudian las agendas de investigación es importante conocer la justificación y el argumento plasmado en cada proyecto como orientador del por qué de la toma de decisión respecto de qué se investiga y su justificación.

La pregunta sobre el para quién se investiga también es pertinente al análisis de las agendas y ha sido particularmente abordada en Latinoamérica en función de definiciones más o menos coloniales y de ciencia asociada a la búsqueda "libre" de la "verdad" y de la supuesta no existencia de fronteras para la indagación mediante métodos aceptados como válidos por el (o los) paradigma (s) vigentes.

En el terreno de la historia de la ciencia se destaca el pensamiento latinoamericano en ciencia y tecnología como ámbito privilegiado donde esta pregunta ha sido relevante y a su vez como potencial inexplorado y desconocido para la propia comunidad científica de la región.

Un aspecto vinculado con lo anterior se emparenta con la idea de compromiso y de engagement (Fals Borda, 1967). La ciencia que se dice orientar por "pura curiosidad" ha servido para colocar un velo sobre las prácticas académicas de los países periféricos: una integración subordinada que poniendo atención en el aspecto cualitativo de la vinculación internacional reconoce aspectos controversiales en la relación entre centro y periferia en cuanto a la definición de agendas de investigación y la división del trabajo científico resultante en la colaboración ha redundado en verdaderas subcontrataciones (Kreimer, 2006). Las revistas académicas que se encuentran en el pujante mercado académico que lucra con un perverso sistema de evaluación que cuenta papers naturaliza el pago por publicar (incluso en revistas de acceso abierto). Fischman (2012) Babbini (2014), Perrotta (2014) y otros han advertido acerca del negocio sobre 
la ciencia que lideran las grandes editoriales que primero se han manifestado contra el acceso abierto pero que en la actualidad han logrado encontrar un nicho de mercado incluso en un movimiento que se pronunciaba por la democratización del conocimiento al menos el producido con financiamiento público6.

Así las agendas se reconocen influenciadas y eso no es perjudicial ni beneficioso, es una verdad negada.

En efecto, la problematización en torno a la definición de agendas de investigación obliga a mirar con más detalle cómo son estas definidas, qué papel juega el contexto y, hacia adentro de la "cocina de la ciencia", qué tipo de vinculaciones-relaciones tienen los científicos con sus colegas de otras latitudes y cómo impacta esto en la elección de temas de investigación.

Nuestros días se caracterizan por tener una ciencia altamente internacionalizada y transnacionalizada (Vessuri, 1983) donde los temas de investigación son definidos por los centros científicos hegemónicos.

Para comprender las prácticas científicas en sociedades periféricas es necesario tener en cuenta la dinámica particular de la ciencia en la escena internacional, en relación (o en oposición) a los factores que operan en el contexto local de desarrollo de las mismas. Cueto (1989) presenta una distinción entre ciencia periférica y ciencia en la periferia. La segunda acepción remite al contexto local en que se produce el conocimiento y fundamentalmente procura resaltar que "no toda la ciencia de los países atrasados es marginal al acervo del conocimiento y que el trabajo científico tiene en estos países sus propias reglas que deben ser entendidas no como síntomas de atraso o de modernidad, sino como parte de su propia cultura y de las interacciones con la ciencia internacional". (Cueto, 1989).

Reconociendo entonces el aspecto dinámico del binomio centro/periferia complejizándolo más allá de la ubicación geográfica del lugar de trabajo o del centro de investigación como así también del país que lo contienen y su relación con los centros económicos del mundo, es posible prestar atención sobre las dinámicas que se dan en las prácticas científicas en el marco de la movilización del conocimiento. Pareciera difícil consolidar algún mecanismo que permita orientar desde la política científica nacional las investigaciones a fin de promover estrategias de uso del conocimiento producido vinculadas a la relevancia social de la producción de los académicos sorteando el dilema definido por la categoría CANA7 (Kreimer y Thomas, 2004).

Pareciera particularmente complejo en este contexto en el que la vinculación y cooperación internacional les son reclamadas a los grupos periféricos (en este sentido la evaluación de la actividad científica juega un rol clave) lograr sortear entonces la subordinación existente. La actividad científica difícilmente pueda pensarse disociada o sin vinculación a nivel internacional, rechazarla sería no reconocer la importancia de las relaciones con la producción de otras latitudes y de la capacidad enriquecedora 
del intercambio. Sin embargo, resulta necesario revisar la forma en la que se dan estas vinculaciones incorporando también como un elemento normativo la relevancia social de la producción académica en la periferia reconociendo que el statu quo de las prácticas en la actualidad constriñe más de lo que habilita a los científicos (y otros actores implicados) a promover sus prácticas poniendo el acento en lo local, quizás retomando el planteo prospectivo de Vessuri sobre aquella "comunidad híbrida" que promueva una "ciencia comprometida con la compleja maraña de constricciones culturales y definiciones políticas que dan forma al ordenamiento de la vida social” (Vessuri, 1983: 69).

\subsection{La industrialización del trabajo científico: la EVALUACIÓN}

La industrialización de las actividades académicas (Musselin, 2007) ha sido tematizada como parte de las actuales condiciones de producción intelectual (Naidorf et al, 2012) y dice responder a la ampliación de la base de individuos que se dedican a actividades científicas en términos comparados con las realidades pretéritas. Asimismo la profesionalización de la ciencia y un modelo no sólo basado en el prestigio sino también en la rentabilidad monetaria ha sido motivo para justificar las medidas de control en una tarea antaño relegada a unos pocos.

Desde la profesionalización (o más bien industrialización) de la actividad académica desde mediados del SXX con la aparición de la bigsciencela evaluación de la actividad académica se instaura como el principal proceso regente en la asignación de recursos para el desarrollo de las actividades de investigación, separándose del pasado idílico en que la evaluación de la ciencia suponía uno de los métodos de contrastación de los criterios de verdad de los enunciados científicos resultando ésta en un tipo específico de capital simbólico: el prestigio académico.

La construcción de nuevos indicadores de prestigio serían medidas principalmente por la indexación de las revistas, es decir qué publicaciones se iban a incluir en el listado de publicaciones que cumplieran determinados criterios, y su clasificación jerárquica según el factor de impacto de cada una.

Una serie de estudios recientes (deRijcke 2016; Whitley et al. 2010, Crespi y Gauna, 2004 y 2005) están problematizando la evaluación, los parámetros de acceso a las carreras científicas, becas de tiempo completo y asignaciones de financiamiento para el desarrollo de la investigación. Enfoques sociológicos sobre los efectos de la de la evaluación sugieren que las prácticas de evaluación no representan ni miden de forma neutral la calidad del trabajo académico sino que además reconfiguran su organización intelectual y social (Dahler-Larsen, 2012).

Adicionalmente, desde las ciencias sociales y humanidades se advierten nuevos matices que complejizan la tarea: los mecanismos de evaluación (y medición) están diseñados tomando más en cuenta los aspectos cuantitativos más usuales en las ciencias naturales, mientras que los tipos de publicación más comunes en ciencias 
sociales y humanidades están más orientados a audiencias locales (Van Leeuwen, 2013), en ocasiones no limitadas al ámbito académico.

\subsection{La utilidad, las usabilidad y el USO del conocimiento científico en el siglo XXI.}

En la actualidad ha tomado especial vigor el debate en torno a los aportes que realizan los científicos a la sociedad, en especial en un contexto de retracción económica que, en línea con la consolidación de modos de organización social que ponderan la eficacia en la asignación de recursos, pugnan por señalar aquellos espacios improductivos a fin de recortarlos.

Este aporte suele volverse inteligible recayendo en el análisis sobre la utilidad social del conocimiento científico, o dicho de otra forma, el uso e impacto que tiene la producción de conocimiento científico en la resolución de problemas de orden social o en la conjugación de demandas sociales.

Más allá de la rendición de cuentas y el tratamiento denigratorio de los grupos concentrados y los gobiernos de derecha que justifican recortes en ciencia con argumentos falaces en torno a la inutilidad de la ciencia, el uso de la ciencia es un tema siempre vigente y controversial. Las respuestas pasadas y los abordajes pretéritos sobre la utilidad y el uso del conocimiento científico son la base para comenzar a abordar el tema pero en el actual contexto requieren una reactualización.

El problema de la utilidad social del conocimiento científico, entendido como uno de los aspectos vigentes de la actividad académica, constituye uno de los grandes temas de investigación de los estudios sociales sobre la ciencia y la tecnología y más generalmente, una de las dimensiones de mayor relevancia para la comprensión del papel desempeñado por los productores de conocimiento científico dentro de una sociedad.

En efecto, en la actualidad, el análisis sobre la utilidad del conocimiento es entendido como una condición socialmente construida en un proceso de interacción en el cual están presentes negociaciones de significados entre diferentes actores (Vaccarezza, 2009; Estébanez, 2007).

De esta forma, cuando se refiere al estudio de la utilidad social del conocimiento científico se requiere identificar entre el tipo de conocimiento que se produce, el tipo de uso posible de conocimiento y las relaciones de recursos que surgen de esos procesos de negociación de significados. Beyer (1997) describe tres tipos de usos del conocimiento científico: la solución de problemas específicos representa un uso instrumental y directo del conocimiento, mientras que su uso para promover la reflexión, la crítica y la conceptualización (uso conceptual) o para sostener y legitimar una idea o posición (uso simbólico) son indirectos. En lo relativo al tipo de conocimiento, es decir, el contenido, la forma, las posibilidades de movilización de lo que se intercambia y transfiere (conocimiento científico, aparatos, técnicas, procesos, "saber hacer") pueden determinar en gran medida los medios o mecanismos de intercambio y transferencia de 
conocimiento a emplear. En última instancia, resulta clave hacer foco sobre el potencial destinatario del conocimiento(Weiss, 1979) puesto que las estrategias que le permitan apropiarse del conocimiento producido no pueden ser escindibles del proceso de producción. Tanto a nivel local como internacional, pareciera existir una deuda por parte de las autoridades institucionales a la hora de incorporar conocimiento científico en su toma de decisión, siendo que: "los resultados muestran que las decisiones políticas a nivel ministerial están principalmente influenciadas por factores políticos y pragmáticos, creencias personales y profesionales y conocimiento local. El papel de la investigación externa se demuestra que es relativamente marginal y se reduce a estudios cuantitativos y evaluaciones de desempeño" (Galway y Sheppard, 2015: 2).

Esperamos que la ciencia, como diría Polanyi (1968), tenga (cumpla) una "función social" y una "utilidad evidente". Siendo que la utilidad es una categoría siempre en suspenso (Vaccarezza, 2009) y solamente podemos hablar de ella mediante una estrategia de seguir al conocimiento producido en su trayectoria por la práctica social, en los sentidos atribuidos, discutidos, de los distintos actores involucrados en su producción, transmisión y uso (Vaccarezza, 2009). De esta manera, una política de ciencia que se proponga establecer una "utilidad de la ciencia" más evidente debería consistir en generar los procesos de interacción y construcción social de significados que se dan entre estos actores: no bastaría abrir una línea de financiamiento para proyectos "aplicados" o "estratégicos", sino acompañar la interacción entre los actores y ayudar a hacer explícito el proceso de construcción de significados posibles de utilidad.

Afirmamos, entonces, que promover (o incluso reclamar) desde la política científica, un mayor compromiso con la utilidad implica, también, dar vuelta el eje de la apelación: supone permitir y facilitar a los grupos sociales el interés y la apropiación consiente de los recursos y prácticas científicas y de la producción científica que gira en torno de ellos (Vaccarezza, 2009).

\section{Conclusiones}

El presente trabajo se propuso recapitular y reformular algunos de los elementos teóricos que sostienen el marco normativo propuesto por la movilización del conocimiento a la hora de considerar y diseñar políticas públicas en materia de producción de conocimiento. En efecto, comprender la incidencia que tienen la priorización de agendas, los mecanismos de evaluación y las definiciones posibles que adoptan la utilidad, uso e impacto de la producción de conocimiento habilita a marcos de acción posibles más complejos pero menos explorados que permitan romper la lógica unidimensional de transmisión o transferencia de conocimiento.

La movilización del conocimiento, en este sentido, pareciera consolidarse como un campo de conocimiento que no solo se proponga indagar y diagnosticar los desafíos 
presentes en el camino hacia la promoción de vías de uso concretas del conocimiento, sino también ofrecer o ensayar respuestas posibles que permitan sortear las trabas existentes en la materia. En cualquier caso, sigue siendo aún un desafío encontrar estrategias que permitan encontrar los interlocutores políticos clave para que estos estudios se consoliden como evidencia que colabore al diseño de políticas públicas donde la movilización del conocimiento se consolide como un ethos ineludible para quienes producen conocimiento científico. Por cierto, la publicación de este número especial da cuenta de ello.

\section{Referencias Bibliográficas}

Bennet, A., Bennet, D., Fafard, K., Fonda, M., Lomond, T., Messier, L., \&Vaugeois, N. (2007). Knowledge mobilization in the social sciences and humanities. Frost, WV: Mqi Press.

Beyer, J. M. (1997). Research utilization bridging a cultural gap between communities, Journal of Management Inquiry, 6, 17-22.

Burkhardt, H., \&Schoenfeld, A. H. (2003). Improving educational research: Toward a more useful, more influential, and better-funded enterprise. Educational researcher, 32, 3-14.

Cooper, A., \&Shewchuk, S. (2015). Knowledge brokers in education: How intermediary organizations are bridging the gap between research, policy and practice internationally. Education policy analysis archives, 23, 118.

Crespi, G. \&Geuna, A. (2004) The Productivity of Science. SPRU Report prepared for the Office of Science and Technology (OST) of the Department of Trade and Industry (DTI), London. Disponible en: http://www.druid.dk/conferences/summer2004/papers/ds2004-77.pdf

Cueto, M. (2012). Excelencia científica en la periferia. In Lima, G. Dahler-Larsen, P. The Evaluation Society. Stanford, CA: Stanford University Press.

Da Silva, R. S. (2016). A Relevância da Epistemologia para o Pensamento Crítico. Revista Lusófona de Educação, 32, 17-29

Estébanez, M. E. (2007). Ciencia, tecnología y políticas sociales. Ciencia, docencia y tecnología, (34), 13-63.

Fals Borda, O. (1967). Ciencia y compromiso. ECO Revista de la Cultura de Occidente, 16, 2.

Fischman, G. \& Haas, E. (2012). Beyond idealized citizenship education: Embodied cognition, metaphors, and democracy. Review of Research in Education, 36(1), 169-196.

Kochanek, J. R., Scholz, C., \& Garcia, A. J. (2015). Mapping the collaborative research process. Education policy analysis archives, 23, 121.

Kreimer, P. \& Thomas, H. (2004). Producción y uso social de conocimientos. Estudios de sociología de la ciencia y la tecnología en America Latina. Bernal: Universidad Nacional de Quilmes.

Kreimer, P. (2006). ¿Dependientes o integrados? La ciencia latinoamericana y la nueva división internacional del trabajo. Nómadas, 24, 199-212. 
Kreimer, P. (2015). La ciencia como objeto de las ciencias sociales en América Latina: Investigar e intervenir. Cuadernos del Pensamiento Crítico Latino americano CLACSO 27, 1-7.

Levesque, P. (2009). Knowledge Mobilization Works. Disponible en www.knowledgemobilization.net

Levin, B. (2011). Mobilising research knowledge in education. London Review of Education, 9, 15-26.

Llomovate, S., Naidorf, J., \&Pereyra, K. (2009). La universidad cotidiana: experiencias de transferencia universidad-sociedad. Buenos Aires. Eudeba.

Musselin, C. (2007). The transformation of academic work: facts and analysis. Research \& Occasional Paper Series: CSHE.4.07 (1).

Naidorf, J. \& Pérez Mora, R. (Org.) (2012). Las actuales condiciones de producción intelectual en Argentina, Brasil y México. Buenos Aires: Miño y Dávila.

Naidorf, J., \& Perrotta, D. (2015). La ciencia social politizada y móvil de una nueva agenda latinoamericana orientada a prioridades. Revista de la educación superior, 44(174), 19-46.

Nutley, S. \& Walter, I. \& Davies, H. \& T. Huw (2007). Using evidence: How research can inform public services. Bristol: The Policy Press.

Rijcke, S., Wouters, P. F., Rushforth, A. D., Franssen, T. P., \&Hammarfelt, B. (2016). Evaluation practices and effects of indicator use-a literature review. Research Evaluation, 25(2), 161-169.

Scott, J., Jabbar, H., LaLonde, P., DeBray, E., \&Lubienski, C. (2015). Evidence use and advocacy coalitions: Intermediary organizations and philanthropies in Denver, Colorado. Education Policy Analysis Archives, 23(124).

Vaccarezza, L. (2009). Las relaciones de utilidad en la investigación social, Revista Mexicana de Sociología, 71, 133-166.

Van Leeuwen, T. (2013). Bibliometric Research Evaluations, Web of Science and the Social Sciences and Humanities: ¿A Problematic Relationship?Bibliometrie - Praxis und Forschung, 2. Disponible en http://www.bibliometrie-pf.de/article/view/173>

Vessuri, H. (1983). La ciencia periférica. Caracas: Monte Avila.

Weiss, C. (1979). The Many Meanings of Research Utilization, Public Administration Review, 39, 426431.

Whitley, R. (2010). La organización intelectual y social de las ciencias. Bernal: Universidad Nacional de Quilmes.

\section{Endnotes}

1 SSHRC Of Canadá, 19 de agosto de 2016, disponible en: https://goo.gl/nZK1mD

2 La metodología de análisis se realizó vía SCOPUS, las publicaciones fueron seleccionadas por el cumplimiento de dos condiciones: contener la frase "KnowledgeMobilization" en las palabras clave y en el abstract del documento. Resultados disponibles en: www.scopus.com/term/analyzer

3 Y los incluidos en el número especial sobre "Movilización del Conocimiento" de la Revista EPAA, disponible en: http://epaa.asu.edu/ojs/issue/view/vol23 
4 La figura de gestor del conocimiento se ha multiplicado en la última década en carreras de posgrado y en formación financiada por los órganos de política científica así como por los particulares. Referencias a ello podemos encontrar en Brasil, Argentina, Perú, Costa Rica y otros.

${ }^{5}$ Fuente: Kochanek, J. R., Scholz, C., \&Garcia, A. N. (2015). Mapping the collaborative research process. Education Policy Analysis Archives, 23(121).

6 Perrotta, D., Saforcada, F. y Babini, D. (2014) "Política académica y evaluación de las ciencias sociales", presentación en el marco del Encuentro de discusión sobre política académica y evaluación en Ciencias Sociales. Organizado por el Instituto de Estudios y Capacitación de CONADU (IEC-CONADU), junto al Consejo Latinoamericano de Ciencias Sociales - CLACSO y FEDUBA. 26 de agosto de 2014. Buenos Aires, Argentina.

7 Conocimiento Aplicable No Aplicado (CANA) (Kreimer y Thomas, 2004, pp. 121-132).

\section{Judith Naidorf}

Doctora en Educación e Investigadora Adjunta del Consejo de Investigaciones Científicas y Técnicas (CONICET) con sede en el Instituto de Investigaciones en Ciencias de la Educación (IICE) de la Facultad de Filosofía y Letras, Universidad de Buenos Aires (UBA). Email: judithnaidorf@gmail.com

ORCID: 0000-0001-8215-5273

Mauro Alonso

Licenciado en Sociología. Becario Doctoral de la Universidad de Buenos Aires (UBA) con sede en el Centro de Estudios sobre Ciudadanía, Estado y

Asuntos Políticos (CEAP).

Email: mauroralonso@gmail.com ORCID: 0000-0002-8644-2592

\section{Correspondência} Judith Naidorf Instituto de Investigaciones en Ciencias de la Educación (IICE)

Facultad de Filosofía y Letras Universidad de Buenos Aires (UBA)

Puán $4804^{\circ}$ Piso, Of. 440 1406 - Ciudad de Buenos Aires (Argentina)

Data de submissão: Dezembro 2016

Data de avaliação: Janeiro 2017

Data de publicação: Março de 2018 